\title{
Online Tool for Dynamical Heart Rate Variability Analysis
}

\author{
Matti Molkkari, Janne Solanpää, Esa Räsänen \\ Computational Physics Laboratory, Tampere University, Tampere, Finland
}

\begin{abstract}
We present a straightforward yet versatile online tool for rapid heart rate variability (HRV) analysis utilizing stateof-the-art methods. The unique feature of the tool is the dynamical examination of accurate RR interval (RRI) correlations as a function of time. This is accomplished by the recently developed dynamical detrended fluctuation analysis, which determines local scaling exponents continuous in both time and scale. Therefore, the tool is particularly well-suited for studying HRV during highly non-stationary situations, such as physical exercise. To this end, the tool integrates with common sport watches.
\end{abstract}

The tool reveals complex correlations in the RRIs during physical exercise. Different sports at various intensities leave distinct fingerprints in the dynamic correlations. Another key advantage of the temporal fidelity is the possibility to study transient alterations in the HRV that could be indicative of various cardiac diseases.

We have implemented an accessible online tool for HRV analysis that is attractive to both researchers and enthusiasts alike. The tool could accelerate cardiac research by providing a quick visual overview of dynamic HRV measures, and permitting further custom analysis by downloadable raw data.

\section{Introduction}

Heart rate variability reflects the state of the autonomic nervous system and cardiovascular health [1]. Studying the correlations between beat-to-beat (RR) intervals reveals fractal-like behavior that is altered by various factors, such as disease or physical exercise [2,3]. These RRI correlations are commonly studied by detrended fluctuation analysis (DFA) [4]. In some situations, such as during sports, it is desirable to study transient changes in HRV. To this end, a dynamical approach to DFA has been developed, which has been successfully employed in studying RRI correlations during running and different sleep stages $[5,6]$. Here we present an online tool for dynamical HRV analysis that implements the method.

\section{Dynamical HRV Analysis}

Conventionally HRV measures are computed for the complete RR interval time series or in simple nonoverlapping segments. Here, we employ a dynamic segmenting scheme to explore the time-dependence of HRV in detail. It is straightforward to obtain enhanced temporal granularity by permitting the segments to overlap in time. Additionally, the locality of a HRV measure is determined by the time scale over which the measure is computed. Therefore, the dynamical behavior of HRV is revealed by a two-dimensional landscape constructed from a segmentation that is allowed to vary in both time and scale.

It is trivial to compute conventional measures, e.g. SDRR or RMSSD, in such dynamic segments. Hence, our tool focuses on the dynamical implementation of DFA (DDFA). The conventional division into arbitrary short( $\alpha_{1}, 4-16$ beats) and long-scale $\left(\alpha_{2}, 16-64\right.$ beats) scaling exponents [4] does not often fully reflect the fractal scaling properties of the RR intervals [7]. Therefore, we compute individual scaling exponents for each scale to obtain a landscape of scaling exponents $\alpha(t, s)$ as the function of both time and scale. The technical details of the DDFA method are described in Ref. [5].

\section{Online Tool}

The DDFA Online tool is available at https:// ddfa-online.accuqt. com. The tool is freely accessible for everyone. Optional user registration offers additional features, such as the possibility to fetch data from other online services, e.g., from Polar or Suunto web APIs. Registered users may also opt in for permitting the usage of their data for research purposes.

\subsection{Graphical User Interface}

The website of the tool serves as its graphical user interface (GUI). The guiding principle for its design has been simplicity and the ease of use for users without background in the topic. The basic workflow of the tool consists of the following steps:

1. Choose the data to be analyzed. 
2. Choose settings for the analysis. This step is optional, as the default settings are designed to be suitable for most purposes.

3. Study the results of the analysis.

The details of these steps are described below.

\subsubsection{Data Selection}

To familiarize users with the tool, we provide some example data that the users may explore before uploading their own data. The most generic format for providing data is via "comma-separated values" (CSV) files. The first row of these files should contain column headers. The user should then indicate which column contains the RR intervals and denote their units. The user may also choose additional columns to be included, such as time, speed, cadence, longitude, latitude or altitude. Practical data is also automatically computed, e.g., the instantaneous heart rate from the RR intervals, if it is not already present. Arbitrary numerical data associated with the RR intervals may also be included. These supplementary data enable additional analysis as the HRV measures are aggregated over these variables to present the HRV measures as their function. Furthermore, the inclusion of position data allows the visualization of the results on an interactive map.

The tool also supports "Flexible and Interoperable Data Transfer" (FIT) files that are utilized by many wearable devices. These files do not require additional user input, but the differences in the practices of device vendors result in limited support.

\subsubsection{Settings}

The primary settings that control the results of the analysis are:

Dynamic length factor $\left(l_{\mathrm{DDFA}}\right)$, which controls the length of the dynamic segments for each scale $s$ at which the DDFA is performed. The DDFA scaling exponent $\alpha(t, s)$ is computed in segments of length $l_{\mathrm{DDFA}} s$. Hence, this parameter controls the balance between locality and statistical noise in the DDFA results. Generally the choice $l_{\text {DDFA }}=5$ is suitable for HRV analysis [5].

Dynamic smoothness priors detrending is an option for explicit detrending of the RRI data before the analysis. While the DFA method performs intrinsic detrending during the analysis, it is limited to polynomial trends and higher order detrending has issues of its own [5]. Therefore, we employ the conventional smoothness priors detrending [8] by separately detrending the data for each scale $s$ before DDFA. The smoothing parameter is chosen in such a manner that features longer than the scale are considered trends.

The filtering of erroneous RR intervals attempts to automatically remove spurious RRIs by determining whether the values are outliers based on the local median and interquartile range.

\subsubsection{Results}

The results of the analysis may be studied from various plots that are described below. The interactive plots may be zoomed and panned to study the details in long RRI recordings. The user may also download the raw data of the results for custom offline analysis.

Time series plot visualizes the RRI data given as input, along with all the other chosen data columns.

$D D F A$ results are shown as a landscape of color-coded DDFA exponents $\alpha(t, s)$. Additionally, a column from the time series and a conventional HRV measure may be chosen as overlay layers for reference. An example is shown in Fig. 1, along with the description of the plot controls.

Aggregated DDFA results illustrate the relationship between the DDFA scaling exponent and some variable of interest, such as the RR interval, heart rate or cadence, which was included as an additional column in the time series. This is accomplished by averaging the variable within the dynamic segments, binning the averages, and then averaging the DDFA scaling exponents within each bin. Additionally, a similarly aggregated conventional HRV measure may be chosen as an overlay layer for comparison.

Map view shows the traveled route color coded by the DDFA scaling exponent for a chosen scale on an interactive map.

\subsection{Application Programming Interface}

In addition to the easy-to-use GUI, the application programming interface (API) is publicly exposed to permit programmatic access for performing computations and fetching results. The current version of the API is available at https: //ddfa-api.accuqt.com/v1/ with the following endpoints for unregistered users:

The data endpoint is utilized for uploading user data for analysis, and it returns an identifier that is utilized for interacting with the data.

The compute endpoint is invoked to perform the actual computations for the given data identifier. It returns either the computation results or an identifier to fetch the results later, depending whether it is invoked asynchronously.

The results endpoint is called to fetch the results of the computations based on the identifier returned by the compute endpoint.

As the tool undergoes continuous development, the complete and up-to-date documentation for the API is available at https://ddfa-online.accuqt. com/docs/api. 


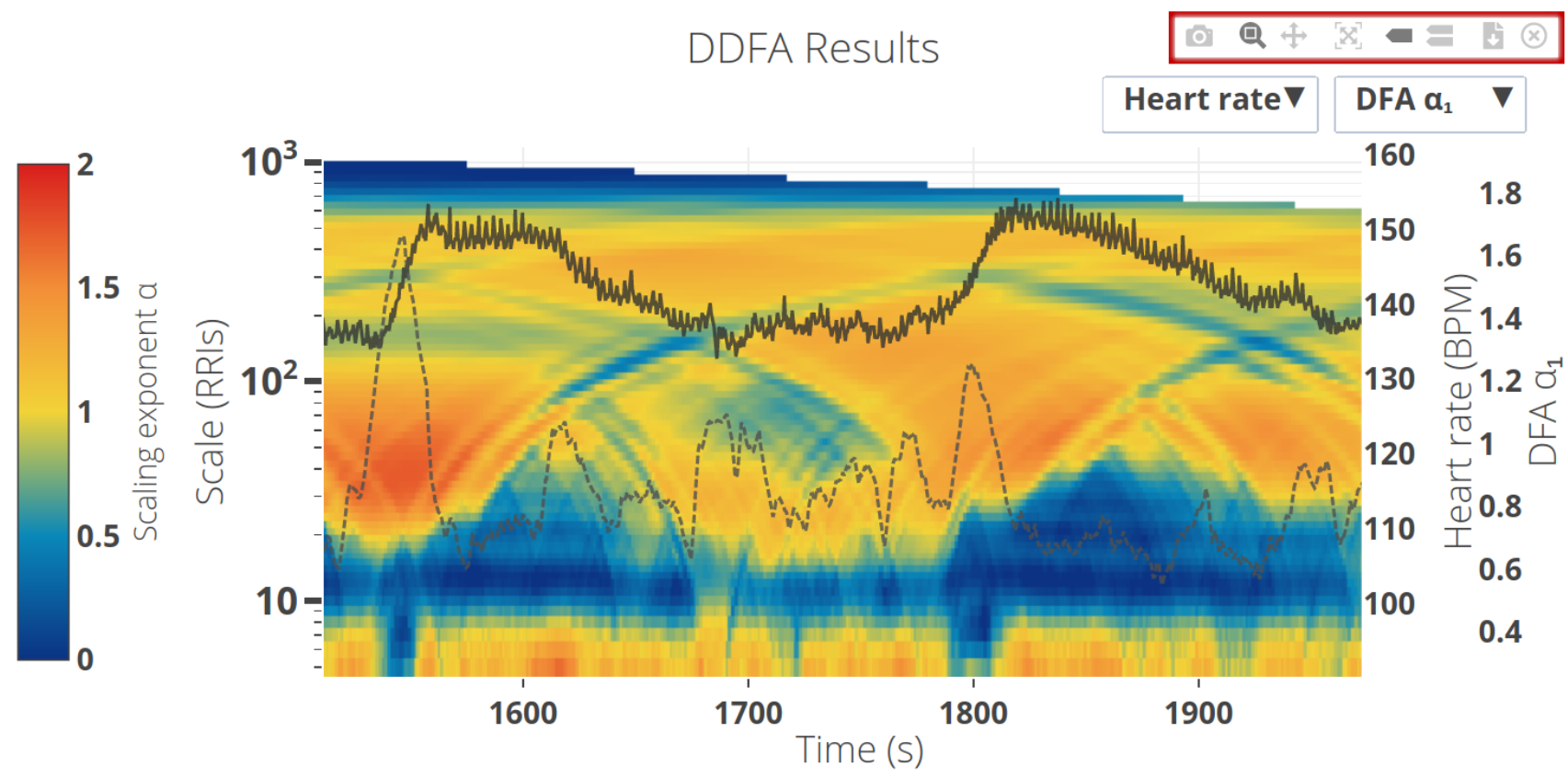

Figure 1. A zoomed-in example of DDFA results with the heart rate (solid line) and the conventional short-scale DFA $\alpha_{1}$ (dashed line) as overlays. The plot controls, encircled in red, are from left to right: i) save the plot as an image, ii) zoom tool, iii) pan tool, iv) autoscale, v) show closest data, vi) compare data, vii) download raw data, viii) close the plot. The dropdown menus $(\boldsymbol{\nabla})$ are utilized for choosing the data for the overlay layers.

\section{Examples}

An example of DDFA results is shown in Fig. 1 for a volunteer performing interval training on an indoor rowing machine. The zoomed-in example spans two intervals with increased intensity and their cooldown periods, as indicated by the overlaid heart rate. There is a strong band of anticorrelations $(\alpha<0.5)$ at scales of roughly 10-20 beats, which is less prominent during the cooldown period. Notably, the conventional DFA $\alpha_{1}$ does not capture the anticorrelated behavior, as it is based on a linear fit within the 4-16 beat range.

The analysis also employs the dynamic smoothness priors detrending. Its significance becomes apparent when looking at the onsets of the high-intensity intervals during the sharp rise in the heart rate. The DDFA $\alpha$ shows anticorrelations that now span all the way to the shortest scales. This is logical, as the strong modulation in the heart rate with periodicity of about 10 beats is suppressed. Instead, there are pairs of shorter and longer beats superimposed on the trend that dominates the modulation. In contrast, DFA $\alpha_{1}$ is highly elevated, which is an artifact due to the trend in the heart rate that is not sufficiently managed by the linear detrending of DFA-1. We hypothesize that the modulation and the resulting short-scale correlations are caused by effects arising from the periodic rowing motion with a certain stroke rate.
It is instructive to study whether there is a relationship between the DDFA $\alpha$ and the heart rate. This is illustrated in the aggregated DDFA results of Fig. 2(a) where the DDFA $\alpha$ is plotted as a function of the heart rate. The anticorrelations clearly become stronger and span wider range of scales as the heart rate increases. While not as prominent, the correlated band at the shortest scales also has a dilating tendency as the heart rate increases. For comparison, the same person performed a $52 \mathrm{~km}$ skiing marathon in Fig. 2(b). The most glaring difference is the lack of a correlated band at the shortest scales. The appearance of short-scale anticorrelations during high intensity exercise and their broadening with increasing heart rate appears to be universal, but the details of the correlation landscapes vary, which is also consistent with the results during running [5].

As an additional example, the DDFA $\alpha$ as a function ST deviation for a patient suffering from ST episodes is shown in Fig. 3, together with pRR20. While there appears to be deviations towards the extremal values, this isolated example is not to be understood as a definite result, but instead as an inspiration for future research.

\section{Discussion and Conclusions}

By employing DDFA, the tool discovers complex fractal correlations that remain hidden from classical methods. 


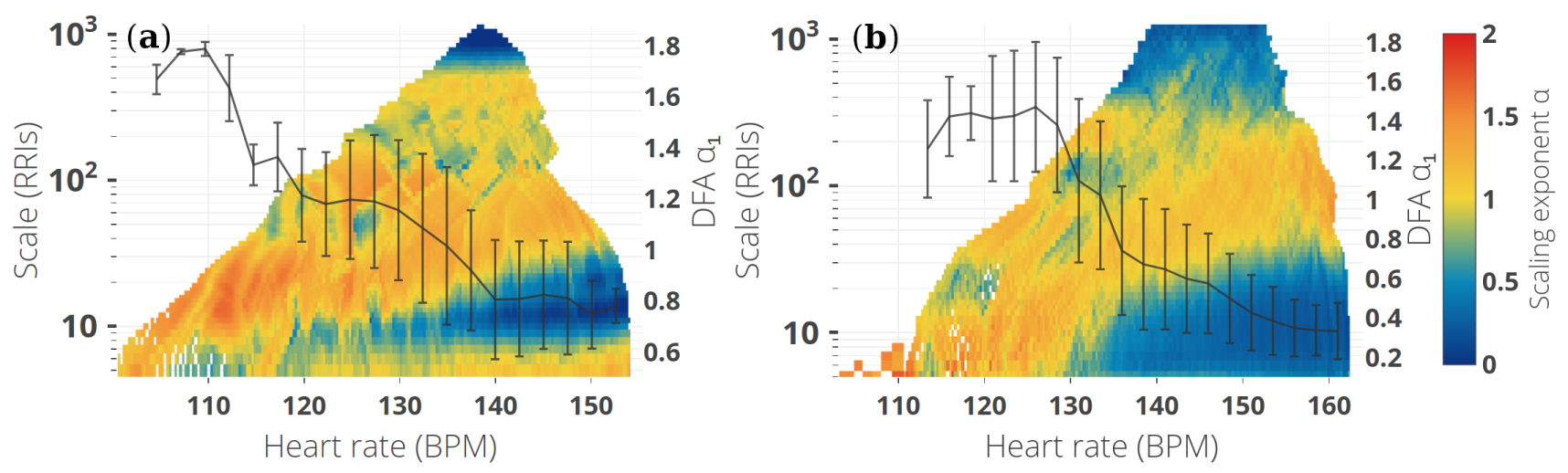

Figure 2. Comparison of aggregated DDFA results as a function of the heart rate during indoor rowing (a) and skiing (b). The conventional short-scale DFA $\alpha_{1}$ is overlaid on the results with the error bars indicating its standard deviation within the heart rate bins. Dynamic smoothness priors detrending was performed prior to DDFA.

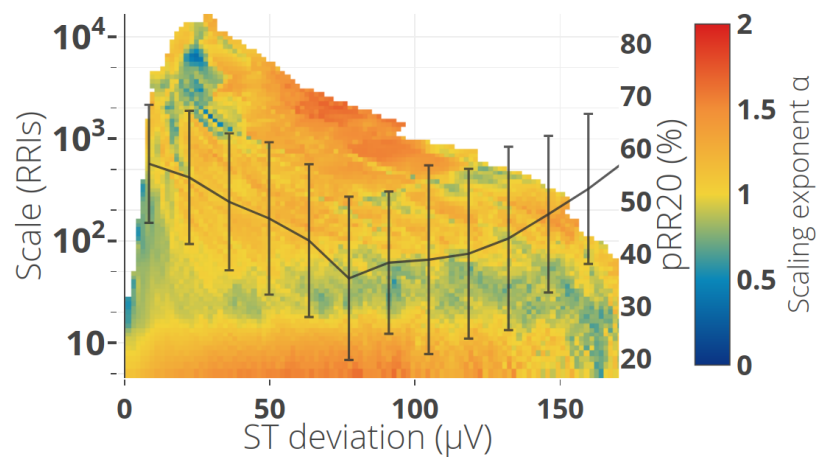

Figure 3. Aggregated DDFA results as a function of ST deviation for a patient suffering from ST episodes. Similarly aggregated pRR20 measure with its standard deviation is overlaid for comparison. Data from PhysioNet Long Term ST Database.

The greatest potential of the tool is achieved in highly nonstationary situations or when transient effects are important. Regardless, the DDFA method could be advantageous even in situations where the data does not exhibit appreciable time-dependence on average, as the local nature of the method permits the extraction of scale-dependent distributions of $\alpha$ from a single time series. This has been demonstrated in sleep monitoring, where also the variance in the correlations is a distinct feature during different sleep stages [6].

This paper has been motivated by the desire to communicate the possibilities of the DDFA method for a broader audience. This goal is facilitated by the online tool presented herein, which provides an accessible avenue for experimenting with state-of-the-art dynamical HRV analysis that is immediately available. There exists an almost endless supply of data in the modern world with a multitude of different prospective research directions. The tool could accelerate cardiac research by providing a quick visual overview of dynamic HRV measures, and permitting further custom analysis by downloadable raw data.

\section{References}

[1] Malik M, et al. Heart rate variability: Standards of measurement, physiological interpretation, and clinical use. European Heart Journal 1996;17(3):354-381.

[2] Goldberger AL, et al. Fractal dynamics in physiology: Alterations with disease and aging. Proceedings of the National Academy of Sciences 2002;99(suppl 1):2466-2472.

[3] Gronwald T, Hoos O. Correlation properties of heart rate variability during endurance exercise: A systematic review. Annals of Noninvasive Electrocardiology 2020; 25(1):e12697.

[4] Peng C, et al. Quantification of scaling exponents and crossover phenomena in nonstationary heartbeat time series. Chaos 1995;5(1):82-87.

[5] Molkkari M, et al. Dynamical heart beat correlations during running. Scientific Reports 2020;10(1):13627.

[6] Molkkari M, et al. Non-linear heart rate variability measures in sleep stage analysis with photoplethysmography. Computing in Cardiology 2019;46:287.

[7] Molkkari M, Räsänen E. Robust estimation of the scaling exponent in detrended fluctuation analysis of beat rate variability. Computing in Cardiology 2018;45:219.

[8] Tarvainen MP, et al. An advanced detrending method with application to HRV analysis. IEEE Transactions on Biomedical Engineering 2002;49(2):172-175.

Address for correspondence:

Matti Molkkari

Tampere University, computational physics laboratory

Korkeakoulunkatu 7, 33720 Tampere, Finland

matti.molkkari@tuni.fi 